IP Periodica Polytechnica Chemical Engineering

61(1), pp. 39-50, 2017

DOI: $10.3311 /$ PPch.10273

Creative Commons Attribution (i)

RESEARCH ARTICLE

\section{Search for the Origin of Discrepancies in Osmotic Measurements of the PNIPAM - Water System}

\author{
Enikő Manek ${ }^{1}$, Etelka Tombácz $^{2}$, Erik Geissler ${ }^{3}$, Krisztina László $^{1 *}$
}

Received 16 November 2016; accepted 04 January 2017

\begin{abstract}
Major, still unelucidated, inconsistencies exist in the literature among measurements of the thermodynamic properties of poly(N-isopropylacrylamide) (PNIPAM) solutions and gels. This paper looks for evidence of intrinsic ionic behaviour in cross-linked PNIPAM homopolymer hydrogels synthesized in water under standard conditions. Systematic measurements are made of the swelling and osmotic properties of lightly cross-linked PNIPAM hydrogels, as well as of their potentiometric titration and DSC response, over a wide range of $\mathrm{pH}$ and ionic strength conditions, in order to distinguish the effects of the latter two parameters on putative intrinsic ions. The intrinsic ion content of the gel is found to be vanishingly small, and consequently unlikely to be the source of the divergences among past measurements. By contrast, a major finding of this study is that comparison of the present results with the literature reveals that frustrated equilibrium can be a source of substantial discrepancies.
\end{abstract}

\section{Keywords}

PNIPAM, osmotic pressure, DSC, ionic strength, $\mathrm{pH}$

\footnotetext{
${ }^{1}$ Department of Physical Chemistry and Materials Science, Faculty of Chemical Technology and Biotechnology, Budapest University of Technology and Economics, H-1521 Budapest, Hungary

${ }^{2}$ Department of Physical Chemistry and Materials Science, University of Szeged, H-6720 Szeged, Hungary

${ }^{3}$ Laboratoire Interdisciplinaire de Physique, Université Grenoble Alpes and CNRS, 38402 Grenoble cedex, France

${ }^{*}$ Corresponding author, e-mail: klaszlo@mail.bme.hu
}

\section{Introduction}

Poly(N-isopropyl-acrylamide) (PNIPAM) is a temperature sensitive water soluble polymer that displays lower critical solution behaviour: above a certain temperature it phase separates. Hydrogels made from this material correspondingly exhibit a volume phase transition (VPT), in which the gel collapses at a temperature $T_{V P T} \sim 34{ }^{\circ} \mathrm{C}$, expelling most of the solvent. Below $T_{V P T}$ PNIPAM gels are hydrophilic, and adopt their swollen configuration. The compulsive quest to exploit this property in innumerable applications, including those with bio-engineering aims such as vectors for controlled drug delivery, scaffolds in tissue growth and engineering, etc. $[1,2]$, has diverted attention from our disturbingly inadequate knowledge of the material properties of PNIPAM solutions and gels.

It has recently been pointed out by Halperin et al. [3] that, among the immense body of experimental investigations into the phase diagram of aqueous solutions of PNIPAM that have been conducted since the pioneering work of Heskins and Guillet [4], agreement remains at best qualitative. Measurements of the osmotic pressure, another thermodynamic parameter, are, by contrast, rare [5, 6]. Nonetheless, in this case also, agreement is merely qualitative, the numerical results being mutually incompatible. Such disorder, which should be a legitimate source of concern for the scientific discipline, suggests that the PNIPAM system may be more complex than previously believed.

Part of the observed experimental variation may stem from the inherent difficulty of preparing monodisperse samples of thermosensitive polymers. Another "usual suspect" source of inconsistencies is chain branching, but in the polymerization of PNIPAM this seems unlikely to play a significant role. Differences between measurements can also arise from variations of tacticity due to preparation at different temperatures or in solvents of different polarity [7, 8]. It is conceivable, for example, that the size of the statistical segment length depends on the extent of syndio-, iso- or atactic regions that develop during polymerization. A further, rarely discussed, source of variation is frustrated thermal equilibrium, or memory effect: in the present article we illustrate this phenomenon with an example. In the case of cross-linked PNIPAM hydrogels, yet 
another possible cause arises if polymer chains acquire partial ionic character during free radical polymerisation in water. In the literature this eventuality is frequently cited, but only inferred [9-12]. Clear evidence, either for or against, is lacking. Schild, in an admirably incisive review of the properties of PNIPAM both in hydrogels and in aqueous solution, explicitly refers to this possibility, opining that "the amount of ionic groups introduced by the initiator and differences among the various experimental techniques employed for observing the transition account for the discrepancies among the various research groups" [13]. While different experimental techniques may yield different types of average in a measurement, it is well established that ionic groups certainly do change the physical properties of the PNIPAM homopolymer [7, 8, 14, 15]. In this text, for the case of gels, we use the term homopolymer to refer exclusively to the network chains, ignoring the crosslinker. The principal purpose of the present investigation is to circumscribe the causes of the observed diaspora of experimental results by examining one of the above possible sources, namely the ionic group hypothesis. To this end we try to evaluate the ionic content of PNIPAM hydrogels synthesized under normal conditions, according to standard recipes with ammonium persulphate as initiator [16, 17], but avoiding deliberate functionalization of the polymer [18].

The presence of ionic groups is in fact difficult to ascertain, as the task of distinguishing the effects of intrinsic ionic groups on the properties of a polymer from those of attendant ions that surround polar groups is not always clear-cut. For this reason we employ a variety of observational methods. The paper is organised as follows. First, as it is a sensitive indicator of intrinsic ionic content, we consider the osmotic pressure of gels and recall the method by which it is determined. Then the sample preparation and the experimental methods used to characterise the gels are defined, including potentiometric titration, swelling measurements and DSC, conducted systematically under different conditions of $\mathrm{pH}$, ionic strength and buffer solution. A comparison is made between the osmotic pressure of the uncross-linked polymer in pure water and that of the gels, as deduced from the swelling measurements. This is followed by an investigation of the variation of the osmotic pressure of the gels as a function of added salt. The response of PNIPAM gels to ions follows closely that of uncross-linked PNIPAM solutions [19-21]. The findings are discussed in comparison with polyelectrolyte gels, a class of polymers that exhibits analogous volume phase transition behaviour in the presence of salt. Relevant background information and measurements are included in the Supplementary Information.

The literature on PNIPAM contains an immense amount of information on the effects of ionic salts and $\mathrm{pH}$ on its transition properties under different conditions. A secondary motivation for this work is that the many of the actual and potential applications of PNIPAM hydrogels synthesized under standard conditions, e.g., in body fluids, various co-solutes under different ionic conditions, call for a systematic picture that discriminates between the influence of ion concentration and that of $\mathrm{pH}$ on the swelling and the osmotic properties.

\section{Theoretical Background}

The ability of gels to swell and deswell is governed by the free energy landscape, and is expressed through a measurable quantity, the osmotic pressure. The amount by which a gel swells in a given solvent is determined by the balance between the osmotic pressure $\Pi$, which expands the network, and the volume elastic modulus $G_{V}$, which limits extension of the chains. The analysis used in the following adopts the Flory-Rehner scheme [22], as employed by Horkay and Zrinyi [23], according to which at swelling equilibrium with the free solvent

$$
\Pi=G_{V}
$$

In lightly cross-linked gels such as those described here the volume elastic modulus $G_{V}$ has been shown to be numerically equal to the shear elastic modulus $G$ [24].

For a given gel composed of independent Gaussian chains [25],

$$
G=G_{0} \varphi^{1 / 3}
$$

where $\varphi$ is the polymer volume fraction and $G_{0}$, the value of the elastic modulus extrapolated to $\varphi=1$, depends only on the cross-link density and on the absolute temperature $T$. Moreover, according to scaling theory and experimental observations $[26,27]$, the osmotic pressure $\Pi$ obeys a power law dependence on $\varphi$ of the form

$$
\Pi=A \varphi^{n}
$$

where, in the case of excluded volume statistics, $n$ is close to 9/4. If the polymer contains uncompensated ionic groups, the resulting electrostatic repulsive interaction enhances the value of $A$ and causes greatly increased swelling of the gel.

Thus, for a given gel at swelling equilibrium $\varphi_{\mathrm{e}}$ with the solvent,

$$
A=G_{0} \varphi_{\mathrm{e}}^{-23 / 12}
$$

For the present purposes it is assumed that for gels in the swollen state if the solvent is modified, either through the $\mathrm{pH}$ or by addition of salt, the strength of the excluded volume, i.e., the prefactor $A$, may change, but not the nature of the excluded volume statistics, as expressed through the exponent $n$. Furthermore, in the region where the network chains behave according to Gaussian statistics, $G_{0}$ is invariant, and the value of $A$ in Eq. (4) therefore depends only on the polymer volume fraction at equilibrium swelling $\varphi_{\mathrm{e}}$. This latter assumption may become questionable close to the transition temperature $T_{V T P}$, where chain association can take place, but at the lower temperatures considered here, it is probably an acceptable 
approximation. For consistency, in what follows, we replace the polymer volume fraction by the mass concentration $c=\rho \varphi$, where $\rho=1.115 \mathrm{~g} / \mathrm{mL}$ is the density of the dry PNIPAM [28]. This substitution, which is important in comparing experimental results, implies a redefinition of the prefactor $A$. To avoid the use of clumsy units we merely state "where $c$ is in $\mathrm{g} / \mathrm{mL}$ ".

In contrast to gels, in uncross-linked polymer solutions the osmotic pressure can be expressed as the sum of two terms,

$$
\Pi=A_{1} R T c+A_{2} c^{n}
$$

where $A_{1}=1 / M_{n}, R$ being the gas constant, $T$ the absolute temperature, and $M_{n}$ the (number average) mass of the dissolved polymer molecules. In dilute solutions, where the osmotic pressure is simply proportional to the number of dissolved molecules, the first term dominates. The second term in Eq. (5) is the scaling expression that replaces the second and higher order terms in the traditional virial expression for the osmotic pressure [29], and where, in the semi-dilute concentration range $(c \leq 0.1-0.2 \mathrm{~g} / \mathrm{mL})$, the exponent adopts the value $n=9 / 4$. The osmotic pressure measurements of Nagahama et al. [6] in solutions of uncross-linked PNIPAM, on being interpolated to $20{ }^{\circ} \mathrm{C}$, yield for Eq. (5)

$$
\Pi=0.006 c+3.73 c^{9 / 4} \mathrm{MPa}
$$

with $c$ in $\mathrm{g} / \mathrm{mL}$ (see Appendix, Figs. A1, A2). The non-aqueous synthesis route and careful sample preparation employed by these authors minimized ionic impurities, thus providing a benchmark for osmotic pressure data in aqueous solutions of PNIPAM.

The first term in Eq. (6), which arises from the translational freedom of the polymer chains, has no equivalent in gels. However, comparison of the value of $A$ in Eq. (4) for a gel with that of $A_{2}$ for a reference uncross-linked polymer solution in Eq. (6) not only is an important indicator of the presence of electrostatic interactions in the gel, but could also provide a measure of the effect of changes in the solvent composition upon the strength of the excluded volume interaction.

\section{Experimental}

\subsection{Materials}

$N$-isopropylacrylamide (NIPAM) (>99\%) from Aldrich and $N, N, N^{\prime}, N^{\prime}$-tetramethylethylenediamine (TEMED) (99\%), supplied by Fluka, were used without further purification. Hydrochloric acid $(\mathrm{HCl})$ (analytical reagent $=\mathrm{AR}$ ), potassium hydroxide $(\mathrm{KOH})(\mathrm{AR})$, sodium hydroxide $(\mathrm{NaOH})$, calcium chloride $\left(\mathrm{CaCl}_{2}\right)(\mathrm{AR})$ and sodium tetraborate (AR) were purchased from Merck, $N, N$ '-methylenebisacrylamide (BA) (99\%), ammonium persulphate (APS) (99\%), acetic acid (AR), disodium phosphate $\left(\mathrm{Na}_{2} \mathrm{HPO}_{4}\right)(\mathrm{AR})$, disodium phosphate dodecahydrate $\left(\mathrm{Na}_{2} \mathrm{HPO}_{4} \cdot 12 \mathrm{H}_{2} \mathrm{O}\right)(\mathrm{AR})$ and imidazole (AR) from Sigma-Aldrich, and potassium chloride ( $\mathrm{KCl})(\mathrm{AR})$, disodium phosphate monohydrate $\left(\mathrm{Na}_{2} \mathrm{HPO}_{4} \cdot 2 \mathrm{H}_{2} \mathrm{O}\right)$ (AR), boric acid (AR), phosphoric acid (AR) and citric acid (99\%) from Reanal.

\subsection{Synthesis of PNIPAM gels}

PNIPAM hydrogel films of thickness $3 \mathrm{~mm}$ were synthetized from NIPAM monomers and BA cross-linker in the molar ratio $[\mathrm{NIPAM}] /[\mathrm{BA}]=150$. APS was used as initiator in the ratio $[\mathrm{NIPAM}] /[\mathrm{APS}]=34.2$, and the reaction was catalysed by TEMED in the ratio [NIPAM]/[TEMED] $=112$. The role of TEMED is to adjust the $\mathrm{pH}$, and thus stabilise the sulphate free radical [30]. Polymerization took place at $20{ }^{\circ} \mathrm{C}$. Gel samples were dialyzed in doubly distilled water to remove unreacted compounds. For swelling measurements gel films were cut into disks (diameter $7 \mathrm{~mm}$ ), then dried and stored above concentrated sulphuric acid. For calorimetric measurements dried gel disks were powdered to particles of size $0.2-1 \mathrm{~mm}$. The elastic moduli of the samples were measured as reported earlier [31].

\subsection{Buffers}

The $\mathrm{pH}$ sensitivity of the swelling degree was measured on gel disks in three types of aqueous buffer solution in the $\mathrm{pH}$ range 3-9. Phosphate buffers (Table S1, Supplementary Information) were chosen because of their wide relevance in biomedical systems. The commonly used Britton-Robinson buffers are also investigated (Table S2) [32]. Britton-Robinson buffers with constant $\mathrm{pH}$ (4.5) with various ionic strengths are also examined in order to eliminate the effect of $\mathrm{pH}$. The ionic strength (0.045-1.045 M) was set with $\mathrm{KCl}$ (Table S3). Conversely, to eliminate the influence of ionic strength, citrate buffers of different $\mathrm{pH}$ but constant ionic strength $(0.15 \mathrm{M})$ were investigated (Table S4).

\section{Methods}

\subsection{Swelling experiments}

For the swelling measurements dry disks were equilibrated at $20.0 \pm 0.2{ }^{\circ} \mathrm{C}$ in various aqueous solutions for 1 week. The equilibrium swelling ratio $\left(1 / \varphi_{\mathrm{e}}\right.$, where $\varphi_{\mathrm{e}}$ is the equilibrium polymer volume fraction) was determined from the mass balance as:

$$
\frac{1}{\varphi_{\mathrm{e}}}=1+\frac{\left(m_{\text {gel,swollen }}-m_{g e l, d r y}\right) \rho}{m_{g e l, d r y}}
$$

where $m_{\text {gel, dry }}$ is the mass of dry and $m_{\text {gel,swollen }}$ is the mass of equilibrated gel disks, and the density of water is taken to be $1 \mathrm{~g} / \mathrm{mL}$.

\subsection{Differential scanning microcalorimetry (DSC)}

DSC measurements were performed on a MicroDSCIII apparatus (SETARAM). The PNIPAM samples were ground to powder to reduce retardation effects from diffusion. Unless otherwise stated, samples incubated in the aqueous solutions were heated from 10 to $40{ }^{\circ} \mathrm{C}$ at scanning rate $\mathrm{d} T / \mathrm{d} t=0.02{ }^{\circ} \mathrm{C} / \mathrm{min}$. 
This slow scanning rate was chosen to minimise kinetic effects. The onset temperature $T_{\text {onset }}$ was defined as the intersection of the baseline and the tangent at the first peak inflection point. Reproducibility of $T_{\text {onset }}$, was $0.1-0.3{ }^{\circ} \mathrm{C}$. The endothermic enthalpy values $\Delta H$ were obtained from the peak integrals with a standard error of $5-7 \%$. The entropy values $\Delta S$ reported here are defined by

$$
\Delta S=\frac{\Delta H}{T}
$$

\subsection{Potentiometric titration}

The acid-base properties of PNIPAM hydrogel were studied by continuous potentiometric titration in the $\mathrm{pH}$ range of 3 to 10 in $\mathrm{CO}_{2}$-free media on a laboratory-developed system. The $\mathrm{NaCl}$ background electrolyte concentrations were $0.01,0.1$ and $1 \mathrm{M}$, respectively. The initial $\mathrm{pH}$ was measured before titration. At each titration point the equilibrium of acid-base consumption was defined by the $\mathrm{pH}$ settling criterion $\leq 0.0005 \mathrm{pH} / \mathrm{s}$. Surface excess amounts of $\mathrm{H}^{+}\left(n_{\mathrm{H}^{+}}^{\sigma}\right)$ and $\mathrm{OH}^{-}\left(n_{\mathrm{OH}_{-}}^{\sigma}\right)$ were calculated from the electrode output signal. The specific net proton surface excess amount $\left(n^{\sigma}=n_{\mathrm{H}^{+}}^{\sigma}-n_{\mathrm{OH}_{-}}^{\sigma}\right)$ for dilute solution adsorption $[33,34]$ was derived directly from the initial and equilibrium concentrations of the solute at each point of the titration and plotted as a function of the equilibrium $\mathrm{pH}$. The reversibility of the titration was tested in a cycle of forward and backward titrations from the immersion $\mathrm{pH} 5.5$, increasing to $\mathrm{pH} 10$, then descending to $\mathrm{pH} 3$, and finally returning to the immersion $\mathrm{pH}$ 5.5. Titrations reported here were performed at $25^{\circ} \mathrm{C}$.

\section{Results and discussion}

The shear elastic moduli of two separately prepared fully swollen samples measured at $20^{\circ} \mathrm{C}$ were respectively $G=0.83$ $\pm 0.04 \mathrm{kPa}\left(1 / \varphi_{\mathrm{e}}=37.1\right)$ and $G=0.87 \pm 0.04 \mathrm{kPa}\left(1 / \varphi_{\mathrm{e}}=35.7\right)$. Within experimental error, these results are indistinguishable. From Eqs. (1)-(3), these values yield for the osmotic pressure in the gel

$$
\begin{aligned}
\Pi_{\text {gel }} & =0.83 \times(37.1 / \rho)^{9 / 4} c^{9 / 4} \mathrm{kPa} \\
& =(2.2 \pm 0.1) c^{9 / 4} \mathrm{MPa}
\end{aligned}
$$

with $c$ expressed in $\mathrm{g} / \mathrm{mL}$.

Dynamic light scattering yields an independent estimate of the osmotic pressure in these gels [31]. The intensity of light scattered by the osmotic concentration fluctuations in a gel is defined by the Rayleigh ratio

$$
R_{\theta}=4 \pi^{2} k_{B} T c^{2} n_{0}^{2}\left(\frac{d n_{0}}{d c}\right) /\left(M_{o s} \lambda^{4}\right)
$$

where $k_{B}$ is the Boltzmann constant, $T$ the absolute temperature, $n_{0}=1.334$ the refractive index of the gel, $\mathrm{d} n_{0} / \mathrm{d} c=0.167 \mathrm{~mL} / \mathrm{g}$ is the refractive index increment with respect to water [35], and

$$
M_{o s}=c \frac{\partial \Pi}{\partial c}+\frac{4 G}{3}
$$

is the longitudinal osmotic modulus that governs osmotic plane waves in gels [36]. In Eq. (10), $\lambda=6.32810^{-5} \mathrm{~cm}$ is the wavelength of the incident light in vacuo. For a PNIPAM gel swollen to equilibrium with pure water at $20{ }^{\circ} \mathrm{C}$ with swelling ratio $1 / \varphi=35.7$, it was found that $R_{\theta}=1.3310^{-4} \mathrm{~cm}^{-1}$. With the contrast factor in Eq. (10) for the PNIPAM-water system, this yields for the longitudinal osmotic modulus

$$
M_{o s}=2.72 \mathrm{kPa}
$$

From Eq. (11) it follows that

$$
M_{o s}=n \Pi_{\mathrm{gel}}+4 G / 3
$$

With $n=9 / 4$, and the equilibrium swelling condition, $\Pi_{\text {gel }}=G$, we find

$$
M_{o s}=(43 / 12) \Pi_{\mathrm{gel}}
$$

Finally, the dynamic light scattering method thus yields

$$
\Pi_{\text {gel }}=1.85 c^{9 / 4} \mathrm{MPa}
$$

In view of the difference in measurement technique, the agreement between the two independent estimates in Eqs. (9) and (15) is acceptable and confirms that the osmotic pressure pre-factor is appreciably lower in the gel than in the uncrosslinked solution (Eq. (6)). We recall, however, that experimental observations on a variety of polymer gels have shown that, while the exponent $n$ remains the same, the pre-factor $A$ of the osmotic pressure in the gel is consistently lower than in the uncrosslinked solution [37-39]. This difference, attributed to a decrease in the effective polymer concentration due to immobilisation of network chains in the vicinity of the cross-links, amounts to a reduction of $30 \%-50 \%$ in the value of $A$. Conversely, if incompletely screened ionic groups are present, the excluded volume interaction increases. In such cases, uncross-linked polymer solutions do exhibit a substantial increase in their osmotic prefactor $A_{2}$, with no change in the value of $n[40,41]$.

From the comparison between the values of $A$ in the PNIPAM gels and that of $A_{2}$ in Eq. (6) for uncross-linked solutions it can be concluded that, if ionic groups are present in the gels investigated here, their contribution to the excluded volume interaction is small.

An important extension of this discussion arises from a comparison between the present findings and those of Shibayama and Tanaka [5], which provides new insight into the PNIPAM system. The present gels, as well as those of previous work by our group [31] in which the cross-link density was varied, were prepared at $20{ }^{\circ} \mathrm{C}$. The power law dependence on concentration of the shear modulus and the Rayleigh ratio were found to be consistent with the predictions of scaling theory under excluded volume conditions, namely $n \approx 9 / 4$ and $n-2 \approx 1 / 4$, respectively [31]. By contrast, the investigation of ref. 5 found that at $20^{\circ} \mathrm{C}$ the power law variation of $M_{o s}$ is much stronger, with $n>3$. This discrepancy originates from the method of preparation. In ref. 5 a master gel 
was first synthesized at $20^{\circ} \mathrm{C}$ at fixed cross-link content [NIPAM] $/$ $[\mathrm{BA}]=45$. This was then deswollen to different degrees by heating to various temperatures closer to $T_{V P T}$, whereupon specimens of the appropriate diameter were cut from the master gel. These were then placed in the scattering cells out of contact with surplus water and brought to the assigned temperature of measurement. The substantial difference observed in the scaling laws resulting from this procedure indicates that, without contact with surplus solvent, chain associations and folding that occur when PNIPAM gels are brought close to $T_{V P T}$ are not reversible. This phenomenon, analysed in more detail in the Appendix, is a sign of frozen conformations, or frustrated equilibrium, to which the PNIPAM system appears to be susceptible, and which could be a major cause of the observed experimental discrepancies.

\subsection{Acid and base solutions}

Information on the intrinsic ionic content of the gels is found from potentiometric titration. The potentiometric titration curve calculated from the $\mathrm{H}^{+} / \mathrm{OH}^{-}$balance in Fig. 1 demonstrates that PNIPAM is sensitive to $\mathrm{pH}$. Starting from the immersion $\mathrm{pH} 5.5$ up to $\mathrm{pH} 10$, then down to $\mathrm{pH} 3$ and returning to $\mathrm{pH} 5.5$, the titration cycle reveals irreversibility. The irreversibility stems from the limited mobility of ions within the swollen gel, especially of negatively charged $\mathrm{OH}^{-}$ions from the base titrant above $\mathrm{pH} 6$, where the gel matrix is polarized negatively (upgoing curves) [42]. At $\mathrm{pH}<5.5$ surface proton excess occurs, due to protonation of polar region of the polymer side group. At higher $\mathrm{pH}$ the negative values generally correspond to proton release or to binding of hydroxyl ions. In this case the $\mathrm{pH}$ response indicates that the basic character of the polar region is weak. The curve is asymmetric, base consumption being much larger than proton excess in the corresponding $\mathrm{pH}$ region. This asymmetry is also an indicator of preferential affinity of the PNIPAM gel for anions. At the highest $\mathrm{pH}$ values $(\mathrm{pH}>13)$, chemical degradation takes place [43]. These observations bear no hallmark of intrinsic ionic behaviour. They are, on the contrary, consistent with recent work that highlights the important role played in the transition by local interactions between anions and the amide groups in the PNIPAM chain [16, 44-46].

Numerous investigations have examined the question of pH sensitivity of PNIPAM hydrogels [47-53], but these works refer mainly to copolymer systems. Of particular interest is the study of Hirotsu et al. [14], which showed that at neutral $\mathrm{pH}$, NIPAM-acrylic acid copolymer gels undergo a discontinuous transition as a function of temperature above a certain acrylic acid content. For the PNIPAM gels described here, the transition is continuous [54]. This condition sets an upper limit for their intrinsic ionic content of about $0.1 \%$. Figure 2 shows the equilibrium swelling degree $\varphi_{0} / \varphi_{e}$, as well as the onset temperature $T_{\text {onset }}$, as a function of $\mathrm{pH}$ under different solvent conditions. In salt-free conditions (i.e., no added salt) the shape of the swelling curve $\varphi_{0} / \varphi_{\mathrm{e}}$ mimics the potentiometric titration curve of Fig. 1. The maximum in $\varphi_{0} / \varphi_{\mathrm{e}}$ around $\mathrm{pH} 3$ is the consequence of induced polyelectrolyte behaviour associated with protonation of the NIPAM polar group, as mentioned above. In the acidic region below $\mathrm{pH} 3, \varphi_{0} / \varphi_{\mathrm{e}}$ decreases almost linearly with decreasing $\mathrm{pH}$ in response to the increasing ionic strength $(\mathrm{HCl}$, ionic strength range 0.001-1 M). Conversely, in the range $\mathrm{pH} 11-13(\mathrm{KOH}$, ionic strength range $0.001-1 \mathrm{M})$, $\varphi_{0} / \varphi_{\mathrm{e}}$ increases linearly with increasing $\mathrm{pH}$. This behaviour is the signature of increasing hydrolysis at high $\mathrm{pH}$. As with other hydrolysed gels of the acrylamide family, these networks swell by several orders of magnitude when placed in pure water, even to the point of rupture. At pH 13.5 and 14 the ionic strength of the solution causes the gel to collapse. To the naked eye, however, the gels remain intact. The onset temperature, $T_{\text {onset }}$, is practically independent of $\mathrm{pH}$ in the range $3 \leq \mathrm{pH} \leq 10$. Outside this range, $T_{\text {onset }}$ decreases, almost certainly also due to the accompanying increase in ionic strength.

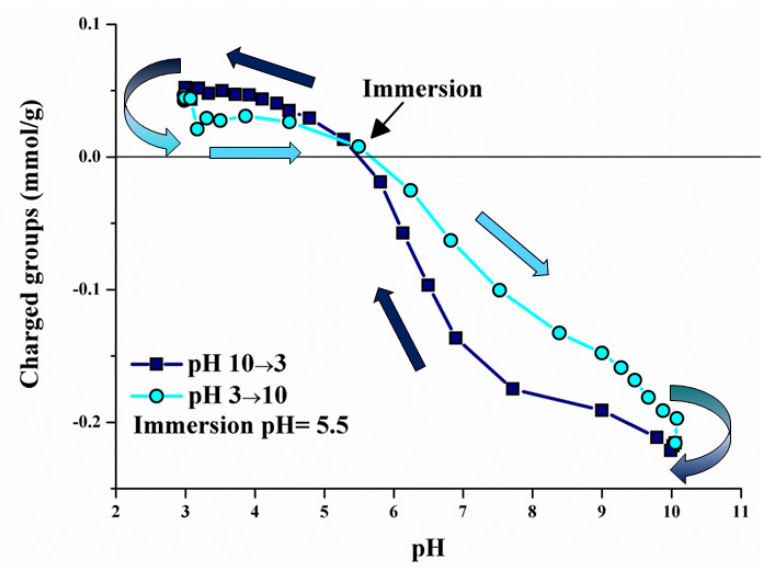

Fig. 1 Potentiometric titration cycles of PNIPAM hydrogel at $25^{\circ} \mathrm{C}$ in $0.01 \mathrm{M}$ $\mathrm{NaCl}$ background electrolyte concentration, starting from immersion $\mathrm{pH} 5.5$.

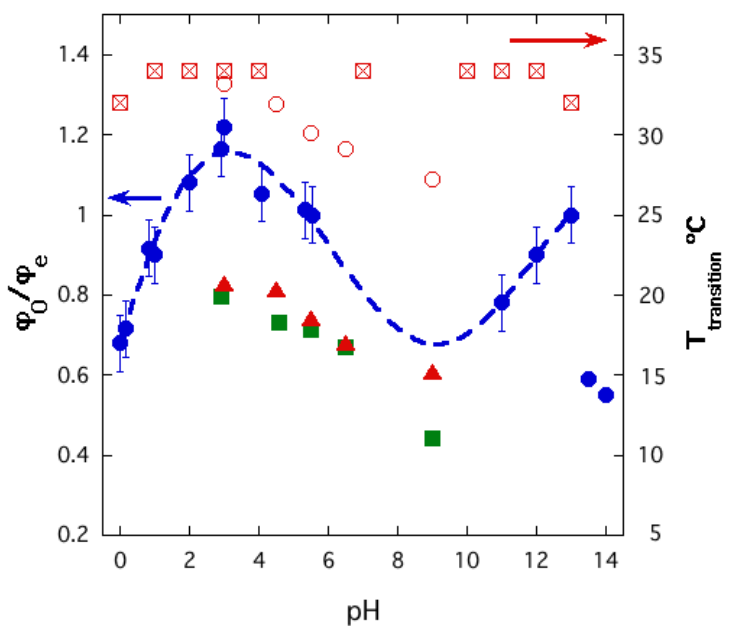

Fig. 2 Dependence of equilibrium swelling degree $\varphi_{0} / \varphi_{\mathrm{e}}$ (left hand axis) and $T_{\text {onset }}$ (right hand axis) at $20{ }^{\circ} \mathrm{C}$ on $\mathrm{pH}$. Solid circles: $\varphi_{0} / \varphi_{\mathrm{e}}$ with no added salt, $\mathrm{pH}$ being defined only by $\mathrm{HCl}$ or $\mathrm{KOH}$ (dashed line: polynomial fit to data, excluding points at $\mathrm{pH}>13)$, solid triangles: $\varphi_{0} / \varphi_{\mathrm{e}}$ with phosphate buffer, solid squares: $\varphi_{0} / \varphi_{\mathrm{e}}$ with Britton-Robinson buffer. The ionic strength in these buffers is listed in Tables S1 and S2 (Supplementary Information). $\bigotimes: T_{\text {onset }}$ with either $\mathrm{HCl}$ or $\mathrm{KOH}$ alone, $\mathrm{O}: T_{\text {onset }}$ with phosphate buffer. 
Figure 2 also illustrates how, when buffer solutions are used to modify the $\mathrm{pH}$, the swelling ratio decreases markedly with respect to the salt free condition, and that $T_{\text {onset }}$ decreases with increasing $\mathrm{pH}$. The response of the gels to buffer solutions (see Supplementary Information) also highlights the importance of the background electrolyte.

The DSC response of PNIPAM samples in acidic solutions for the range $0 \leq \mathrm{pH} \leq 3$ with no added salt, and in basic solutions in the range $11 \leq \mathrm{pH} \leq 14$, is shown in Fig. 3. At the acidic end of the range $T_{\text {onset }}$ decreases linearly with ionic strength $I$, in agreement with observations in the literature [55]. At $\mathrm{pH}=0$, the shape of the transition broadens, indicating a change in the network chains. This change is reversible. At high $\mathrm{pH}$, the shape and the position of the transition peak also vary, but more strongly. The hydrolysis of the polymer chains is irreversible. The enthalpy $\Delta H$ and corresponding entropy $\Delta S$ of the endothermic transition in aqueous solutions of $\mathrm{HCl}$ and $\mathrm{KOH}$ are practically independent of $\mathrm{pH}$ (Table 1).

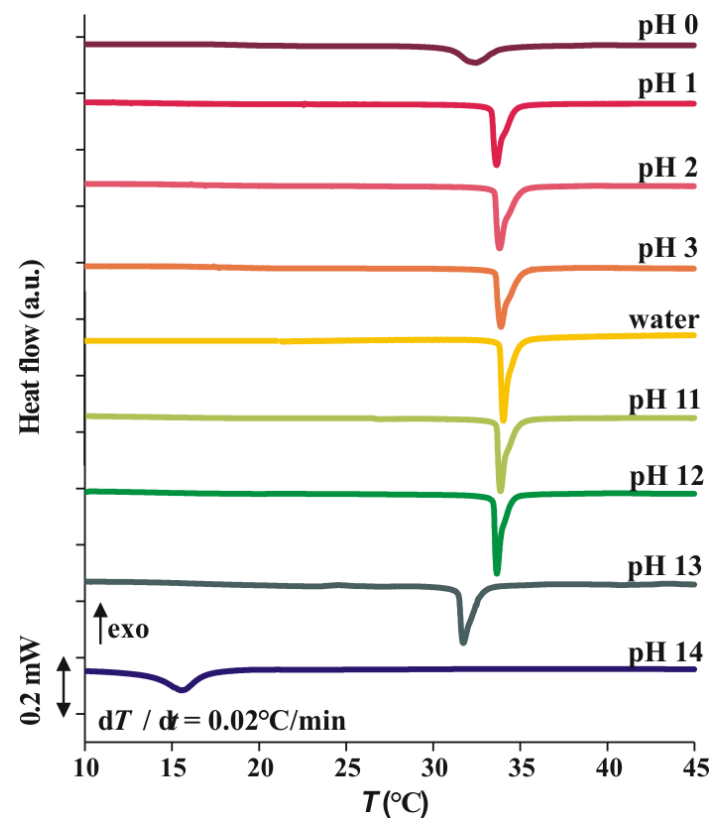

Fig. 3 DSC response of PNIPAM at various $\mathrm{pH}$ set by either $\mathrm{HCl}$ or $\mathrm{KOH}$ solutions.

Table 1 Enthalpy and entropy of the transition in aqueous solutions of $\mathrm{HCl}$ and $\mathrm{KOH}$ (for the $\mathrm{pH}$ interval see Fig. 2)

\begin{tabular}{lcc}
\hline & $\Delta H$ & $\Delta S$ \\
& $\mathrm{~J} / \mathrm{g}_{\text {dry gel }}$ & $\mathrm{J} / \mathrm{g} \mathrm{K}$ \\
\hline $\mathrm{H}_{2} \mathrm{O}$ & $67 \pm 5.9$ & $0.22 \pm 0.02$ \\
$\mathrm{HCl}$ & $60.0 \pm 4.9$ & $0.21 \pm 0.01$ \\
$\mathrm{KOH}$ & $63.2 \pm 2.7$ & $0.20 \pm 0.02$ \\
\hline
\end{tabular}

\subsection{Effect of ions}

The sensitivity of PNIPAM to various anions in the Hofmeister series [56] is well known [21, 46]. Table 2 lists the effect of cations from various alkali and alkali earth metal chlorides on $T_{\text {onset }}$ and on $\Delta H$ and $\Delta S$ of the PNIPAM gel phase transition. The decrease in $T_{\text {onset }}$ due to cations follows the same order as the Hofmeister series, $\mathrm{H}^{+}<\mathrm{Na}^{+}=\mathrm{K}^{+}<\mathrm{Mg}^{2+}<\mathrm{Ca}^{2+}<$ $\mathrm{Sr}^{2+}$. The position and the shape of the phase transition peak of PNIPAM samples determined from the DSC response in aqueous $\mathrm{KCl}$ solutions, display no perceptible trend as a function of ionic strength in the range $0 \leq I \leq 1 \mathrm{M}$ at $\mathrm{pH}$. As also found by other investigators $[16,17,55], T_{\text {onset }}$ decreases linearly with $I$ to a high degree of precision (Fig. S1, Supplementary Information). Within experimental error, the enthalpy and entropy of the transition are independent of ionic strength.

Table 2 Onset temperatures of PNIPAM gels in $1 \mathrm{M}$ alkali and alkaline earth metal chloride solutions

\begin{tabular}{llllllll}
\hline & Water & $\mathrm{HCl}$ & $\mathrm{NaCl}$ & $\mathrm{KCl}$ & $\mathrm{MgCl}_{2}$ & $\mathrm{CaCl}_{2}$ & $\mathrm{SrCl}_{2}$ \\
\hline$T_{\text {onset }}\left({ }^{\circ} \mathrm{C}\right)$ & 33.6 & 30.9 & 20.6 & 20.6 & 19.9 & 18.6 & 14.5 \\
\hline $\begin{array}{l}\Delta H \\
\left(\mathrm{~J} / \mathrm{g}_{\text {drygel }}\right)\end{array}$ & $67 \pm 5.9$ & 74 & 74 & $69.8 \pm 5.6$ & 77 & 71 & 70 \\
\hline $\begin{array}{l}\Delta S \\
\left(\mathrm{~J} / \mathrm{g}_{*} \mathrm{~K}\right)\end{array}$ & $0.22 \pm 0.02$ & 0.24 & 0.25 & $0.24 \pm 0.02$ & 0.26 & 0.24 & 0.24 \\
\hline
\end{tabular}

The effect of the ionic salts $\mathrm{KCl}$ and $\mathrm{CaCl}_{2}$ on the swelling degree and the osmotic pressure of the gels is shown in Fig. 4. On plotting $\varphi_{0} / \varphi_{\mathrm{e}}$ as a function of the ionic strength $I$ of the solution surrounding the gel, with these two salts the VPT occurs at different values of $I$ (Fig. $4 \mathrm{a}$ ). In both cases $\varphi_{0} / \varphi_{\mathrm{e}}$ decreases exponentially with $I$ in the region before the collapse. This response is inconsistent with that of polyelectrolytes, where the osmotic pressure obeys a power law function of ionic strength [57]. With the divalent salt $\mathrm{CaCl}_{2}$, the deswelling exhibits a two-step process that implies partial chain folding, analogous to that observed by Zhang and Cremer with $\mathrm{Na}_{2} \mathrm{SO}_{4}$ [46]. The gel remains transparent as far as $\varphi_{0} / \varphi_{\mathrm{e}} \approx 0.4$.

The corresponding calculated variation of the osmotic pressure pre-factor $A$, defined at each salt concentration by the equilibrium condition Eq. (4), is plotted in Fig. $4 \mathrm{~b}$ as a function of concentration of $\mathrm{Cl}^{-}$ions for both $\mathrm{KCl}$ and $\mathrm{CaCl}_{2}$, rather than as a function of ionic strength $I$, as in Fig. 4a. In this semi-logarithmic representation the straight line behaviour in the swollen state for $\mathrm{KCl}\left(c_{\mathrm{Cl}-}<1 \mathrm{M}\right)$ reproduces the exponential behaviour seen in Fig. 4a. This response differs markedly from that of $T_{\text {onset }}$ (Fig. S1), which decreases linearly with respect either to $I$ or to $c_{\mathrm{Cl}-}$. Fig. $4 \mathrm{~b}$ provides confirmation that it is the anion concentration that determines the VPT, which at $20{ }^{\circ} \mathrm{C}$ takes place at $c_{\mathrm{Cl}-}=1 \mathrm{M}$. With $\mathrm{CaCl}_{2}$ the initial decrease is indistinguishable from that with $\mathrm{KCl}$, but at $c_{\mathrm{Cl}-}>0.5 \mathrm{M}$ it diverges, decreasing approximately as a power law, but, as was observed by Zhang et al. in uncross-linked solutions of PNIPAM [45], in discontinuous steps. This phase of the deswelling trajectory ends at $c_{\mathrm{Cl}}{ }^{-}=1 \mathrm{M}$, the same point of collapse as with $\mathrm{KCl}$. It should 
be borne in mind that the partial chain association and folding that is hypothesized in this intermediate region undermines the assumption that $G_{0}$ is invariant, and hence the numerical values of $A$ in that regime are not reliable. It is also remarkable that in the swollen state, neither response resembles that of a polyelectrolyte solution, where the osmotic pressure varies with ionic strength as $I^{-0.75}$ (broken lines in Fig. 4b) [39, 40]. A striking contrast to this behaviour is provided by polyelectrolyte gels in equilibrium with an infinite bath: the critical ion concentration in the surrounding solution at which the present gels collapse is about three orders of magnitude greater than for polyelectrolyte gels, and the ion exchange capacity is negligible [58]. This comparison again shows that the ionic content of the gel is very much smaller than $1 \%$. These observations are further evidence in favour of the model of Cremer [45, 46], whereby the principal mechanism controlling the VPT is the interaction between anions and the polar groups of PNIPAM.

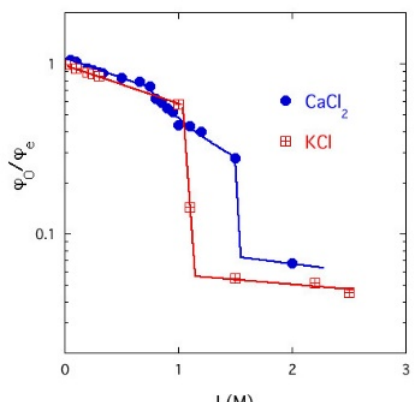

I (M)

a

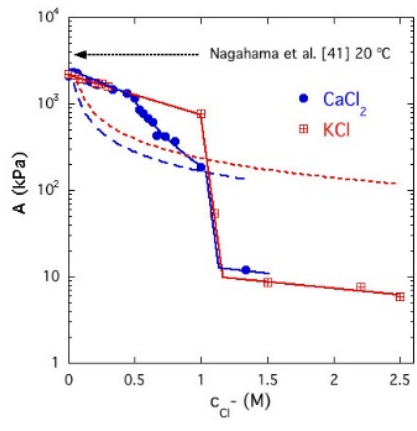

b
Fig. 4 a: Ionic strength dependence of the equilibrium swelling ratio $\varphi_{0} / \varphi_{\mathrm{e}}$ of PNIPA gels in solutions of $\mathrm{KCl}$ (squares) and $\mathrm{CaCl}_{2}$ (circles) at $20^{\circ} \mathrm{C}$.

b: Dependence of the osmotic pressure pre-factor $A=G_{0} \varphi_{\mathrm{e}}^{-23 / 12}$ on the $\mathrm{Cl}^{-}$ anion concentration in aqueous solutions of $\mathrm{KCl}$ (squares) and $\mathrm{CaCl}_{2}$ (circles) at $20{ }^{\circ} \mathrm{C}$. Dashed curves: power law responses $\left(\propto I^{-0.75}\right)$ that prevail in the osmotic pressure of polyelectrolyte solutions as the ionic strength $I$ is varied $[54,55]$. Horizontal arrow: osmotic pressure pre-factor $A_{2}$ for solution of uncross-linked polymer at $20^{\circ} \mathrm{C}$ (Eq. (6)) [6].

With $\mathrm{CaCl}_{2}$, the swelling behaviour in the intermediate region $0.5 \mathrm{M} \leq c_{\mathrm{Cl}-}<1 \mathrm{M}$ is instructive. On one hand, the partial deswelling could be interpreted as evidence that the gel contains a small number, about $0.1 \%$, of ionic groups. It could, on the other hand, arise from a weak interaction of the cation with the amide group. The observation [46] that it also occurs with $\mathrm{Na}_{2} \mathrm{SO}_{4}$ shows that it is unrelated to intrinsic ionic content. These gels thus carry no observable signature of polyelectrolyte systems [59].

The above observations reinforce the notion advanced by Cremer [46] that the mechanism governing the VPT operates through local interactions with hydrophobic side-groups. Such local interactions have already been detected in homopolymer PNIPAM microgels by FTIR and Raman spectroscopy [60], while with foreign probe molecules, such as phenol, they are visualised directly by ${ }^{1} \mathrm{H}$ CRAMPS solid-state NMR [61].

\section{Conclusions}

This article describes a systematic search for evidence of intrinsic ionic behaviour in the response of PNIPAM hydrogels to changes in the $\mathrm{pH}$ and salt conditions of their aqueous environment. Observations are made by potentiometric titration, differential scanning calorimetry, swelling measurements and osmotic pressure. The response of the swelling degree to the ionic salts $\mathrm{KCl}$ and $\mathrm{CaCl}_{2}$ confirms that the VPT of the gel is governed not by the ionic strength, but by the anion concentration in the surrounding solution. The observations fail to detect any intrinsic molar ion content in the gels, and the osmotic response to added salt is inconsistent with that of polyelectrolyte systems. The equilibrium swelling degree depends both on $\mathrm{pH}$ and on the nature of the buffer solution, but in the latter case the effect of the salt is stronger than that of $\mathrm{pH}$. As expected, at high $\mathrm{pH}$ the network chains become increasingly hydrolysed, and ultimately collapse, in response to the elevated anion concentration in the solution. These findings highlight the importance of the background electrolyte.

Although ionic salts affect the osmotic pressure, the phase transition temperature appears to have no direct relationship to the value of the osmotic pressure at the transition threshold. The measurements indicate, on the contrary, that the guest molecules disturb the hydrophilic/hydrophobic balance of PNIPAM through local interactions on the molecular scale.

Our investigation forecloses the idea that charged ionic groups acquired during free radical synthesis of PNIPAM play a significant role in the saga of past experimental inconsistencies. This negative result contrasts with our major finding, obtained by comparing our results with measurements in the literature and described in detail in the Appendix, that frustrated equilibrium in PNIPAM samples can give rise to extremely diverse results.

\section{Acknowledgement}

Support from the Hungarian grant OTKA K115939 (Hungarian Scientific Research Fund) and FP7-PEOPLE2010-IRSES-269267 (Marie Curie International Research Staff Exchange Scheme) project is acknowledged. E. Manek is grateful for the support of an Ernő Pungor Scholarship. We express our gratitude to Avraham Halperin for drawing our attention to this problem and for enlightening discussions, and to E. Wilk for technical assistance.

\section{References}

[1] Galperin, A., Long, T. J., Ratner, B. D. "A degradable, thermo-sensitive poly(N-isopropyl acrylamide)-based scaffold with controlled porosity for tissue engineering applications." Biomacromolecules. 11(10), pp. 2583-2592. 2001. https://doi.org/10.1021/bm100521x

[2] Zhang, X. Z., Lewis, P., Chu C. C. "Fabrication and characterization of a smart drug delivery system: microsphere in hydrogel." Biomaterials. 26(16), pp. 3299-3309. 2005.

https://doi.org/10.1016/j.biomaterials.2004.08.024 
[3] Halperin, A., Kröger, M., Winnik, F. M. "Poly(N-isopropylacrylamide) phase diagrams: fifty years of research." Angewandte Chemie International Edition. (Invited Review). 54(57), pp. 15342-15367. 2015. https://doi.org/10.1002/anie.201506663

[4] Heskins, M., Guillet, J. E. "Solution properties of poly(N-isopropylacrylamide)." Journal of Macromolecular Science: Part A - Chemistry. 2(8), pp. 1441-1455. 1968. https://doi.org/10.1080/10601326808051910

[5] Shibayama, M., Tanaka, T. "Small angle neutron scattering study on poly(N-isopropyl acrylamide) gels near their volume phase transition temperature." The Journal of Chemical Physics. 97, pp. 6829-6841. 1992. https://doi.org/10.1063/1.463636

[6] Nagahama, K., Inomata, H., Saito, S. "Measurement of osmotic pressure in aqueous solutions of poly-(ethylene glycol) and poly(N-isopropylacrylamide)." Fluid Phase Equilibria. 96, pp. 203-214. 1994. https://doi.org/10.1016/0378-3812(94)80096-0

[7] Ray, B., Okamoto, Y., Kamigaito, M., Sawamoto, M., Seno, K., Kanaoka, S., Aoshima, S. "Effect of tacticity of poly(N-isopropylacrylamide) on the phase separation temperature of its aqueous solutions." Polymer Journal. 37, pp. 234-237. 2005. https://doi.org/10.1295/polymj.37.234

[8] Hirano, T., Okumura, Y., Kitajima, H., Seno, M., Sato, T. "Dual roles of alkyl alcohols as syndiotactic-specificity inducers and accelerators in the radical polymerization of $\mathrm{N}$-isopropylacrylamide and some properties of syndiotactic poly(N-isopropyl-acrylamide)." Journal of Polymer Science Part A: Polymer Chemistry. 44(15), pp. 4450-4460. 2006.

https://doi.org/10.1002/pola.21546

[9] Pelton, R. H., Pelton, H. M., Morphesis, A. R., Rowells, L. "Particle sizes and electrophoretic mobilities of poly(N-isopropylacrylamide) latex." Langmuir. 5, pp. 816-818. 1989.

https://doi.org/10.1021/la00087a040

[10] Bischofberger, I., Trappe, V. "New aspects in the phase behaviour of poly-N-isopropyl acrylamide: systematic temperature dependent shrinking of PNiPAM assemblies well beyond the LCST." Scientific Reports. 5, 15520. 2015. https://doi.org/10.1038/srep15520

[11] Clara-Rahola, J., Fernandez-Nieves, A., Sierra-Martin, B., South, A. B., Lyon, L. A., Kohlbrecher, J., Fernandez Barbero A. "Structural properties of thermoresponsive poly(N-isopropylacrylamide)-poly(ethyleneglycol) microgels." The Journal of Chemical Physics. 136, 214903. 2012. https://doi.org/10.1063/1.4723686

[12] Virtanen, O. L. J., Richtering, W. "Kinetics and particle size control in non-stirred precipitation polymerization of $\mathrm{N}$-isopropylacrylamide." Colloid and Polymer Science. 292(8), pp. 1743-1756. 2014. https://doi.org/10.1007/s00396-014-3208-x

[13] Schild, H. G. "Poly(N-isopropylacrylamide): experiment, theory and applications." Progress in Polymer Science. 17, pp. 163-249. 1992. https://doi.org/10.1016/0079-6700(92)90023-R

[14] Hirotsu, S., Hirokawa, Y., Tanaka, T. "Volume-phase transitions of ionized N-isopropylacrylamide gels." The Journal of Chemical Physics. 87, pp. 1392-1395. 1987. https://doi.org/10.1063/1.453267

[15] Karg, M., Pastoriza-Santos, I., Rodriguez-González, B., von Klitzing, R., Wellert, S., Hellweg, T. "Temperature, $\mathrm{pH}$, and ionic strength induced changes of the swelling behavior of PNIPAM-poly(allylacetic acid) copolymer microgels." Langmuir. 24(12), pp. 6300-6306. 2008. https://doi.org/10.1021/la702996p

[16] Geever, L. M., Nugent, M. J. D., Higginbotham, C. L. "The effect of salts and $\mathrm{pH}$ buffered solutions on the phase transition temperature and swelling of thermoresponsive pseudogels based on N-isopropylacrylamide." Journal of Materials Science. 42(23), pp. 9845-9854. 2007. https://doi.org/10.1007/s10853-007-1814-4
[17] Panayiotou, M., Freitag, R. "Influence of the synthesis conditions and ionic additives on the swelling behaviour of thermo-responsive polyalkylacrylamide hydrogels." Polymer. 46(18), pp. 6777-6785. 2005. https://doi.org/10.1016/j.polymer.2005.06.060

[18] Snowden, M. J., Thomas, D., Vincent, B. "Use of colloidal microgels for the absorption of heavy metal and other ions from aqueous solution." Analyst. 118, pp. 1367-1369. 1993.

https://doi.org/10.1039/AN9931801367

[19] Freitag, F., Flaudy, R., Flaudy, G. "Salt effects on the thermoprecipitation of poly(N-isopropylacrylamide) oligomers from aqueous solution." Langmuir. 18(15), pp. 3434-3440. 2002. https://doi.org/10.1021/la0106440

[20] Park, T. G., Hoffman, A. S. "Sodium chloride-induced phase transition in nonionic poly(N-isopropyl-acrylamide) gel." Macromolecules. 26, pp. 5045-5048. 1993. https://doi.org/10.1021/la0106440

[21] Inomata, H., Goto, S., Otake, K., Saito, S. "Effect of additives on phase transition of N-isopropyl-acrylamide gels." Langmuir. 8(2), pp. 687-690. 1992. https://doi.org/10.1021/la00038a064

[22] Flory, P. J., Rehner, J. "Statistical Mechanics of Cross-Linked Polymer Networks II. Swelling." The Journal of Chemical Physics. 11, pp. 521-526. 1943. https://doi.org/10.1063/1.1723792

[23] Horkay, F., Zrinyi, M. "Studies on the mechanical and swelling behavior of polymer networks based on the scaling concept, 4. Extension of the scaling approach to gels swollen to equilibrium in a diluent of arbitrary activity." Macromolecules. 15, pp. 1306-1310. 1982. https://doi.org/10.1021/ma00233a018

[24] Geissler, E., Hecht, A. M., Horkay, F., Zrinyi, M. "Compressional modulus of swollen polyacrylamide networks." Macromolecules. 21, pp. 2594-2599. 1988. https://doi.org/10.1021/ma00186a048

[25] Treloar, L.R.G. In: The physics of rubber elasticity. (3rd edition). Clarendon, Oxford. 1975. https://doi.org/10.1002/pi.4980080107

[26] de Gennes, P.-G. "Scaling concepts in polymer physics." Cornell University Press, Ithaca, New York. 1979. https://doi.org/10.1002/actp.1981.010320517

[27] Adam, M., Delsanti, M. "Dynamical properties of polymer solutions in good solvent by Rayleigh scattering experiments." Macromolecules. 10, pp. 1229-1237. 1977. https://doi.org/10.1021/ma60060a014

[28] László, K., Kosik, K., Rochas, C., Geissler, E. "Phase transition in poly(Nisopropylacrylamide) hydrogels induced by phenols." Macromolecules. 36, pp. 7771-7776. 2003. https://doi.org/10.1021/ma034531u

[29] Flory, P. J. "Principles of Polymer Chemistry." Cornell University Press, Ithaca, New York. 1953.

[30] Cammack, R., Atwood, T., Campbell, P., Parish, H., Smith, A., Vella, F. Stirling. J. (eds.) "TEMED." In: Oxford Dictionary of Biochemistry and Molecular Biology. Oxford. 2006.

[31] László, K., Kosik, K., Geissler, E. High-sensitivity isothermal and scanning microcalorimetry in PNIPA hydrogels around the volume phase transition. Macromolecules. 37(26), pp. 10067-10072. 2004. https://doi.org/10.1021/ma048363x

[32] Mongay, C., Cerda, V. A. "A Britton-Robinson buffer of known ionic strength." Annali di Chimica. 64, 409-412. 1974.

[33] László, K., Kosik, K., Filipcsei, G., Zrínyi, M. "Interaction of non-ionic hydrogels with weak aromatic acids." Macromolecular Symposia. 200, 181-190. 2003. https://doi.org/10.1002/masy.200351018

[34] Everett, D. "Reporting data on adsorption from solution at the solid/solution interface." Pure and Applied Chemistry. 58(7), 967-984. 1986. https://doi.org/10.1351/pac198658070967 
[35] Zhou, S., Fan, S., Au-yeung, S. F. C., Wu, C. "Light-scattering studies of poly(N-isopropylacrylamide) in tetrahydrofuran and aqueous solution." Polymer. 36, pp. 1341-1346. 1995. https://doi.org/10.1016/0032-3861(95)95910-S

[36] Tanaka, T., Hocker, L. O., Benedek, G. B. "Spectrum of light scattered from a viscoelastic gel." The Journal of Chemical Physics. 59, pp. 5151-5159. 1973. https://doi.org/10.1063/1.1680734

[37] Geissler, E., Horkay, F., Hecht, A. M. "Osmotic and scattering properties of chemically cross-linked poly(vinyl alcohol) hydrogels." Macromolecules. 24, pp. 6006-6011. 1991 https://doi.org/10.1021/ma00022a016

[38] Hecht, A. M., Guillermo, A., Horkay, F., Mallam, S., Legrand, J. F., Geissler, E. "Structure and dynamics of a poly(dimethylsiloxane) network: a comparative investigation of gel and solution." Macromolecules. 25, pp. 3677-3684. 1992. https://doi.org/10.1021/ma00040a011

[39] Horkay, F., Burchard, W., Geissler, E., Hecht, A. M. "Thermodynamic properties of poly(viny1 alcohol) and poly(viny1 alcohol-vinyl acetate) hydrogels." Macromolecules. 26, pp. 1296-1303. 1993. https://doi.org/10.1021/ma00058a017

[40] Horkay, F., Basser, P. J., Londono, D. J., Hecht, A. M., Geissler, E. "Ions in hyaluronic acid solutions." The Journal of Chemical Physics. 131(18), 184902. 2009. https://doi.org/10.1063/1.3262308

[41] Horkay, F., Basser, P. J., Hecht, A. M., Geissler, E. "Chondroitin sulfate in solution: effects of mono- and divalent salts." Macromolecules. 45, pp. 2882-2890. 2012. https://doi.org/10.1021/ma202693s

[42] László, K., Tombácz, E., Novák, C. "pH-dependent adsorption and desorption of phenol and aniline on basic activated carbon." Colloids and Surfaces A: Physicochemical and Engineering Aspects. 306(1-3 SPEC. ISS), pp. 95-101. 2007. https://doi.org/10.1016/j.colsurfa.2007.03.057

[43] Smith, R. M., David, E. "The pH-rate profile for the hydrolysis of a peptide bond." Journal of the American Chemical Society. 120(35), pp. 8910-8913. 1998. https://doi.org/10.1021/ja9804565

[44] Benrebouh, A., Avoce, D., Zhu, X. "Thermo- and pH-sensitive polymers containing cholic acid derivatives." Polymer. 42(9), pp. 4031-4038. 2001. https://doi.org/10.1016/S0032-3861(00)00837-5

[45] Zhang, Y. J., Furyk, S., Bergbreiter, D. E., Cremer, P. S. "Specific ion effects on the water solubility of macromolecules: PNIPAM and the Hofmeister series." Journal of the American Chemical Society. 127(23), pp. 14505-14510. 2005. https://doi.org/10.1021/ja0546424

[46] Zhang, Y., Cremer, P. S. "Interactions between macromolecules and ions: the Hofmeister series." Current Opinion in Chemical Biology. 10(6), pp. 658-663. 2006. https://doi.org/10.1016/j.cbpa.2006.09.02

[47] Zhang, J., Chu, L.Y., Li, Y. K., Lee, Y. M. "Dual thermo- and pH-sensitive poly(N-isopropylacrylamide-co-acrylic acid) hydrogels with rapid response behaviors." Polymer. 48(6), pp. 1718-1728. 2007. https://doi.org/10.1016/j.polymer.2007.01.055

[48] Jones, M. S. "Effect of $\mathrm{pH}$ on the lower critical solution temperatures of random copolymers of $\mathrm{N}$-isopropylacrylamide and acrylic acid." European Polymer Journal. 35(5), pp. 795-801. 1999. https://doi.org/10.1016/S0014-3057(98)00066-4
[49] Cai, H., Zhang, Z. P., Ping, C. S., Bing, L. H., Xiao X. Z. "Synthesis and characterization of thermo- and ph- sensitive hydrogels based on chitosan-grafted $\mathrm{N}$-isopropylacrylamide via $\gamma$-radiation." Radiation Physics and Chemistry. 74(1), pp. 26-30. 2005.

https://doi.org/10.1016/j.radphyschem.2004.10.007

[50] Beltran, S., Baker, J. P., Hooper, H. H., Blanch H. W., Prausnitz, J. M. "Swelling equilibria for weakly ionizable, temperature-sensitive hydrogels." Macromolecules. 24, pp. 549-551. 1991. https://doi.org/10.1021/ma00002a032

[51] Brazel, C. S., Peppas, N. A. "Synthesis and characterization of thermoand chemomechanically responsive poly(N-isopropylacrylamide-comethacrylic acid) hydrogels." Macromolecules. 28(24), pp. 8016-8020. 1995. https://doi.org/10.1021/ma00128a007

[52] Krušić, M. K., Filipović, J. "Copolymer hydrogels based on N-isopropylacrylamide and itaconic acid." Polymer. 47(1), pp. 148-155. 2006. https://doi.org/10.1016/j.polymer.2005.11.002

[53] Suzuki, A., Suzuki, H. "Hysteretic behavior and irreversibility of polymer gels by pH change." The Journal of Chemical Physics. 103, pp. 4706-4710. 1995. https://doi.org/10.1063/1.470608

[54] Kosik, K., Wilk, E., Geissler, E., László, K. "Interaction of phenols with thermo-responsive hydrogels." Colloids and Surfaces A: Physicochemical and Engineering Aspects. 319(1-3), pp. 159-164. 2008.

https://doi.org/10.1016/j.colsurfa.2007.07.022

[55] Schild, H. G. D., Tirrell, A. "Microcalorimetric detection of lower critical solution temperatures in aqueous polymer solutions." The Journal of Physical Chemistry. 94, pp. 4352-4356. 1990. https://doi.org/10.1021/j100373a088

[56] Hofmeister, F. "On the understanding of the effects of salts." Archiv for Experimentelle Pathologie und Pharmakologie. 24, pp. 247-260. 1888.

[57] Dobrynin, A. V., Rubinstein, M. "Theory of polyelectrolytes in solutions and at surfaces." Progress in Polymer Science. 30, pp. 1049-1118. 2005. https://doi.org/10.1016/j.progpolymsci.2005.07.006

[58] Horkay, F., Basser, P. J., Hecht, A. M., Geissler E. "Osmotic and SANS observations on sodium polyacrylate hydrogels in physiological salt solutions." Macromolecules. 33, pp. 8329-8333. 2000. https://doi.org/10.1021/ma000972r

[59] Horkay, F. Basser, P. J., Hecht, A. M., Geissler, E. "Effect of calcium/ sodium ion exchange on the osmotic properties and structure of polyelectrolyte gels." Journal of Engineering in Medicine. 229, pp. 895-904. 2015. https://doi.org/10.1177/0954411915602915

[60] Ahmed, Z., Gooding, E. A., Pimenov, K. V., Wang, L., Asher, S. A. "UV resonance Raman determination of molecular mechanism of poly (N-isopropyl-acrylamide) volume phase transition." The Journal of Physical Chemistry B. 113, pp. 4248-4256. 2009. https://doi.org/10.1021/jp810685g

[61] Domján, A., Manek, E., Geissler, E., László K. "Host-guest interactions in poly(N-isopropyl-acrylamide) hydrogel seen by one- and two-dimensional ${ }^{1} \mathrm{H}$ CRAMPS solid-state NMR spectroscopy." Macromolecules. 46(8), pp. 3118-3124. 2013. https://doi.org/10.1021/ma400295a 


\section{Appendix}

\section{Osmotic pressure in uncross-linked PNIPA}

Measurements of the osmotic pressure, $\Pi_{\text {measured }}$ of aqueous solutions of uncross-linked PNIPAM were reported by Nagahama et al. [6] Owing to the polydispersity of the distribution, and also to the associative nature of this particular polymer, it is difficult physically to remove the low molecular weight tail of the distribution. Since at low concentration $c$ this tail yields a contribution proportional to $c$, however, its effect can be corrected quite well by subtracting a constant $A_{1}$ from the values of $\Pi_{\text {measured }} / c R T$. Fig. A1 shows that the osmotic pressure data $\Pi=$ $\left(\Pi_{\text {measured }} / c R T-A_{1}\right) \times c R T$ from Ref. 6 can, to a good approximation, be represented at all the measured temperatures by a power law function of the concentration of the form

$$
\Pi=A(T) c^{n}
$$

with $A_{1}=0.250$ and $n=9 / 4$, and where $A(T)$ is expressed in $\mathrm{kPa}$ and $c$ in $\mathrm{g} / \mathrm{mL}$. These results are consistent with the scaling law prediction for excluded volume conditions, [26] and are also in agreement with experimental observations on other polymer systems.[27]

Shibayama and Tanaka [5] have also reported osmotic observations in aqueous solutions of PNIPAM, using small angle neutron scattering (SANS) to measure the osmotic modulus $K_{\text {os }}$ $=\varphi \partial \Pi / \partial \varphi$. These measurements, performed as a function of both concentration and temperature, showed that $K_{\text {os }}$ is a power law function of the volume fraction $\varphi$, with the form

$$
K_{\mathrm{os}}=B(T) \varphi^{n}
$$

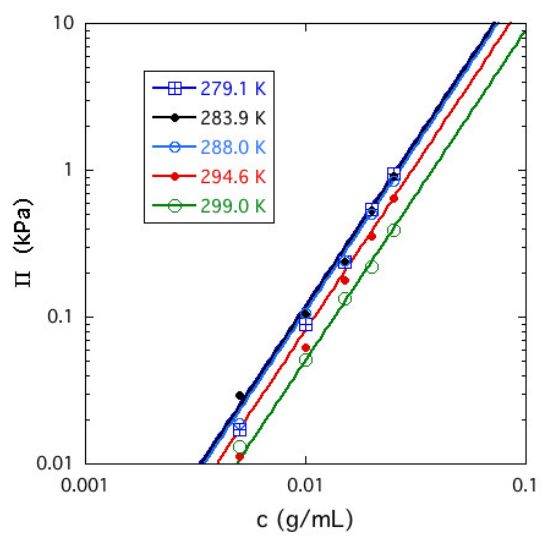

Fig. A1 $\Pi=\left(\Pi_{\text {measured }} / c R T-A_{1}\right) \times c R T$, where $A_{1}=2.50 \times 10^{-6} \mathrm{~mole} / \mathrm{g}$ and $c$ is in $\mathrm{g} / \mathrm{mL}$, expressed as a power law $A_{2}(T) c^{9 / 4}$, from Nagahama et al. [6]. This value of $A_{1}$ corresponds to the molecular weight $M_{n}=400 \mathrm{kDa}$.

Here, as before, $n \approx 9 / 4$. Taking the density of the dry polymer $\rho=1.115 \mathrm{~g} / \mathrm{cm}^{3}$, an expression of the same form as Eq. (A1) is obtained, with

$$
\Pi_{\text {neutr }}=\frac{B(T)}{n \rho^{n}} c^{n}=A_{\text {neutr }}(T) c^{n}
$$

where $A_{\text {neutr }}(T)$ is still expressed in $\mathrm{kPa}$ and $c$ in $\mathrm{g} / \mathrm{mL}$. Fig. A2 compares the direct measurements of the osmotic pressure from ref. 6 and those obtained from the SANS measurements of ref. 5. The difference between these two results is too large to be attributed to the different measuring techniques. It reveals instead a quantitative difference in the material properties of the two PNIPAM samples used in these observations.

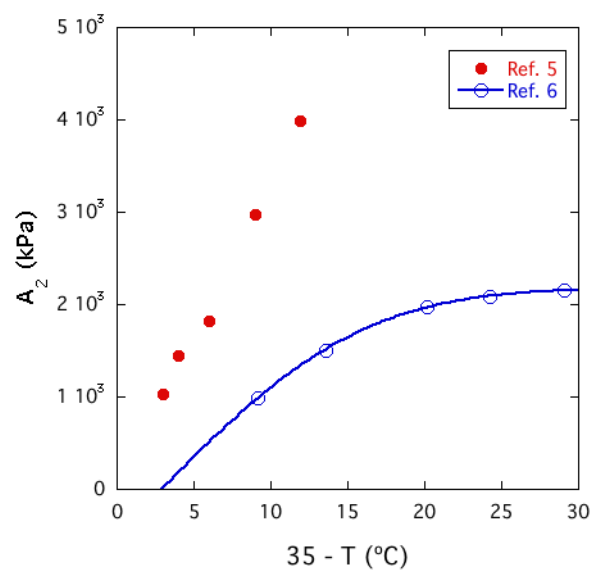

Fig. A2 Open circles: temperature dependence of the power law pre-factor $A_{2}(T)$ from Ref. 6. Continuous curve is the empirical fit $\Pi=2178$ erf $\left[0.0677\left(T_{\mathrm{c}}-T\right)\right]$, with $T_{\mathrm{c}}=305.23 \mathrm{~K}$. Filled symbols: temperature dependence of power law prefactor $A_{\text {neutr }}(T)$ from SANS measurements [5]. In the horizontal axis of the figure $T$ represents the sample temperature in ${ }^{\circ} \mathrm{C}$, where $T=35^{\circ} \mathrm{C}$ defines the origin.

For the cross-linked PNIPAM gels, the neutron scattering measurements of the gel longitudinal osmotic modulus $M_{\mathrm{os}}=K_{\mathrm{os}}+4 \mathrm{G} / 3$ [35] was found in Ref. 5 to vary as $\varphi^{n}$, but in this case $n \geq 3.15$ (Fig. 14 of Ref. 5). This exceptionally strong concentration dependence conflicts with previous results from our group for PNIPAM gels, [30] which exhibited excluded volume behaviour, i.e., $n \approx 9 / 4$. The discrepancy between the two findings can be traced to the different mode of sample preparation. In Ref. 5, as here, the gels were first prepared at $20{ }^{\circ} \mathrm{C}$. To increase the polymer concentration the temperature was then raised to higher values where the gel partially deswelled, at which temperature samples were cut out from the main gel. When the temperature was subsequently lowered again to $20^{\circ} \mathrm{C}$, samples prepared in this way are no longer at swelling equilibrium with the solvent. The results indicate that raising the temperature not only reduces the osmotic pressure but also causes the PNIPAM chains to fold and associate. In the absence of surplus solvent, when the temperature is again reduced, the chains fail to unfold and to exert their full osmotic pressure. This difference between our present results and those of Ref. 5 thus reveals a memory effect in this polymer.

To illustrate the effect of sample preparation on the gels, the data of ref. 5 can be extrapolated to $20^{\circ} \mathrm{C}$. For the same concentration of sample as that reported here $(\varphi=0.028)$, the value of the longitudinal osmotic modulus obtained by neutron scattering is $M_{\mathrm{os}}=0.4 \mathrm{kPa}$, while that obtained in the present samples by dynamic light scattering, without raising the temperature close to $T_{V P T}$, is given by Eq. (12), namely $M_{\mathrm{os}}=2.72 \mathrm{kPa}$. 


\section{Supplementary Information}

(references as in main text)

\section{DSC measurements}

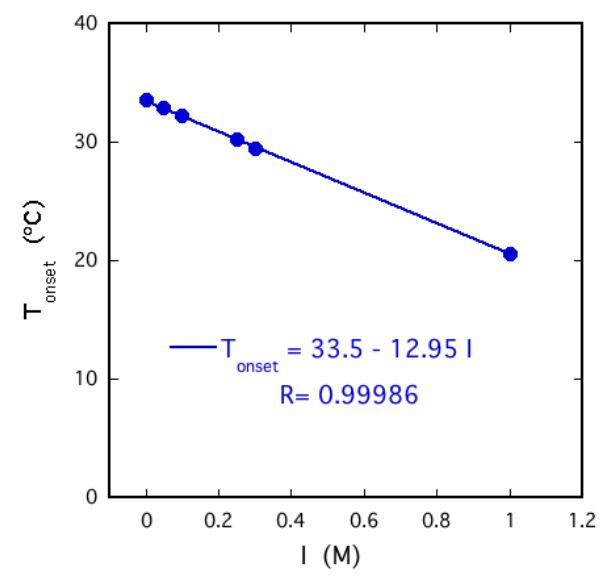

Fig. S1 Dependence of the onset temperature $T_{\text {onset }}$ on the ionic strength of $\mathrm{KCl}$.

\section{Buffer solutions}

Table S1 Composition of the phosphate buffers ${ }^{1}$

\begin{tabular}{llll}
\hline $\mathrm{pH}$ & Materials in $V=1 \mathrm{~L}$ & $\mathrm{pH}$ adjusting component & $I(\mathrm{M})$ \\
\hline 3 & $0.7 \mathrm{~mL} \mathrm{H}_{3} \mathrm{PO}_{4}(85 \%)$ & $\mathrm{NaOH}$ & 0.065 \\
\hline 4.5 & $6.80 \mathrm{~g} \mathrm{KH}_{2} \mathrm{PO}_{4}$ & - & 0.050 \\
\hline 5.5 & $\begin{array}{l}13.12 \mathrm{~g} \mathrm{KH}_{2} \mathrm{PO}_{4}+ \\
1.39 \mathrm{~g} \mathrm{Na}_{2} \mathrm{HPO}_{4} \cdot 12 \mathrm{H}_{2} \mathrm{O}\end{array}$ & - & 0.250 \\
\hline 6.5 & $13.80 \mathrm{~g} \mathrm{NaH}_{2} \mathrm{PO}_{4}$ & $\mathrm{NaOH}$ & 0.120 \\
\hline 9 & $17.40 \mathrm{~g} \mathrm{KH}_{2} \mathrm{PO}_{4}$ & $\mathrm{KOH}$ & 0.280 \\
\hline
\end{tabular}

${ }^{1}$ Sigma-Aldrich, Buffer Reference Center. http://www.sigmaaldrich.com/ life-science/core-bioreagents/biological-buffers/learning-center/bufferreference-center.html\#phosphate/, 2003 [Accessed: 27.07.2016].

Table S2 Composition of Britton-Robinson buffers [32]

\begin{tabular}{llll}
\hline & $V_{\text {acid mixture }}(0.04 \mathrm{M}$ acetic acid, & $V_{\mathrm{NaOH}}(0.2 \mathrm{M})$ & $I(\mathrm{M})$ \\
$\mathrm{pH}$ & $\begin{array}{l}0.04 \mathrm{M} \text { phosphoric acid, } \\
(\mathrm{mL})\end{array}$ & \\
\hline 3 & $1004 \mathrm{M}$ boric acid) $(\mathrm{mL})$ & 17.5 & 0.030 \\
4.5 & 100 & 29.3 & 0.045 \\
5.5 & 100 & 38.6 & 0.055 \\
6.5 & 100 & 47.5 & 0.067 \\
9 & 100 & 68.0 & 0.105 \\
\hline
\end{tabular}

Table S3 Composition of Britton-Robinson buffers pH 4.5 of various ionic strengths [32]

\begin{tabular}{lllll}
\hline & $\begin{array}{l}V_{\text {acid mixture }} \\
\text { (0.04 M acetic acid, } \\
0.04 \mathrm{M} \text { phosphoric acid, } \\
0.04 \mathrm{M} \text { boric acid) }(\mathrm{mL})\end{array}$ & $\begin{array}{l}V_{\mathrm{NaOH}}(0.2 \mathrm{ML}) \\
(\mathrm{mL})\end{array}$ & $m_{\mathrm{KCl}}(\mathrm{g})$ & $I(\mathrm{M})$ \\
\hline 4.5 & 100 & 29.3 & 0 & 0.045 \\
4.5 & 100 & 29.3 & 0.4823 & 0.095 \\
4.5 & 100 & 29.3 & 0.9646 & 0.145 \\
4.5 & 100 & 29.3 & 9.6393 & 1.045 \\
\hline
\end{tabular}

Table S4 Composition of citrate buffers with constant ionic strength $(0.15 \mathrm{M})$

\begin{tabular}{llll}
\hline \multicolumn{4}{c}{ Citric acid buffers ${ }^{1}(V=1 \mathrm{~L})$} \\
\hline $\mathrm{pH}$ & $m_{\text {citric acid anhydride }}(\mathrm{g})$ & $m_{\mathrm{KCl}}(\mathrm{g})$ & $m_{\mathrm{NaOH}}(\mathrm{g})$ \\
\hline 3 & 6.3400 & 9.7100 & 18.9000 \\
\hline 4.5 & 6.3400 & 6.5600 & 49.4000 \\
\hline 5.5 & 6.3400 & 3.3800 & 83.7000 \\
\hline \multicolumn{5}{c}{ Imidazole buffer ${ }^{2}(\mathrm{~V}=1 \mathrm{~L})$} \\
\hline 6.5 & $V_{1 \mathrm{M} \mathrm{HCl}}(\mathrm{mL})$ & $m_{\mathrm{KCl}}(\mathrm{g})$ & $m_{\text {imidazole }}(\mathrm{g})$ \\
\hline \multicolumn{5}{c}{59.1800} & 5.2820 & 6.8080 \\
\hline 9 & $V_{1 \mathrm{M} \mathrm{HCl}}(\mathrm{mL})$ & $m_{\mathrm{KCl}}(\mathrm{g})$ & $m_{\text {borax }}(\mathrm{g})$ \\
\hline & 10.2000 & 7.4570 & 9.5343
\end{tabular}

${ }^{1}$ Sigma-Aldrich, Buffer Reference Center. http://www.sigmaaldrich.com/ life-science/core-bioreagents/biological-buffers/learning-center/bufferreference-center.html\#phosphate/, 2003 [Accessed: 27.07.2016].

${ }^{2}$ Sigma-Aldrich, Buffer Reference Center. http://www.sigmaaldrich.com/ life-science/core-bioreagents/biological-buffers/learning-center/bufferreference-center.html/. [Accessed: 27.07.2016].

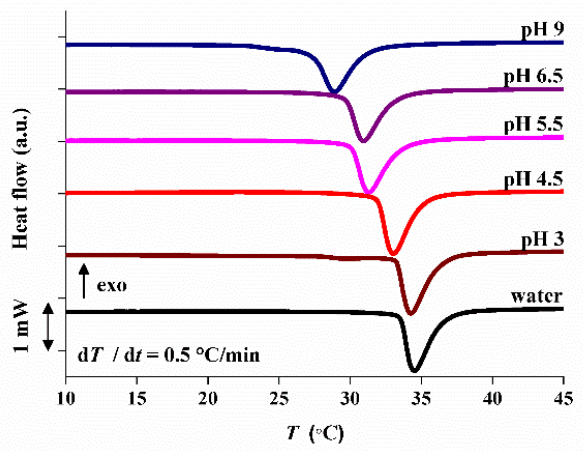

a

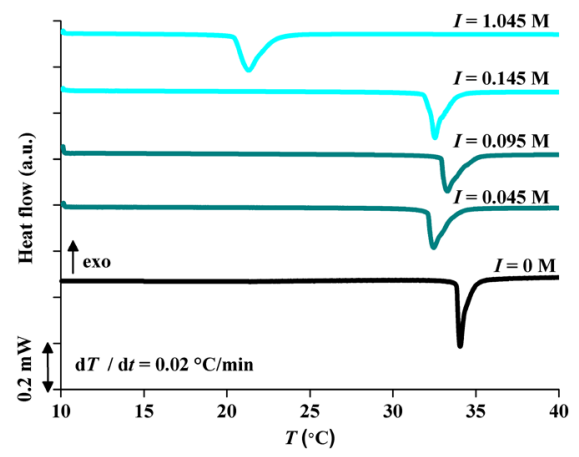

b

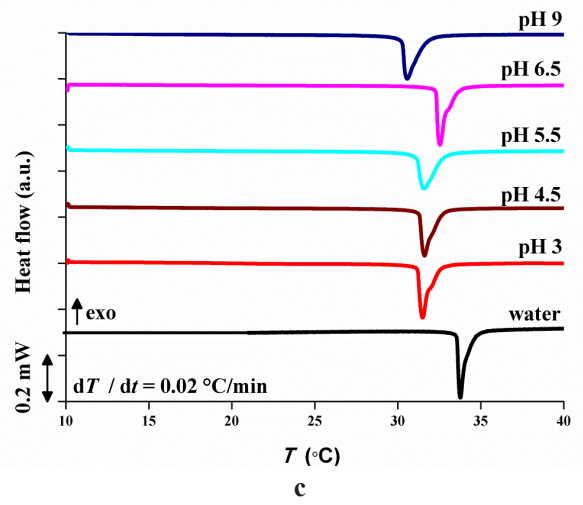

Fig. S2 DSC response of PNIPAM swollen a) in phosphate buffer solutions of different $\mathrm{pH}, \mathrm{b}$ ) in Britton-Robinson buffers $\mathrm{pH} 4.5$ of various ionic strengths and c) in buffers with constant ionic strength $0.15 \mathrm{M}$ 
Figure 2 of the main text shows that with phosphate and Britton-Robinson buffers of different $\mathrm{pH}$, a correlation exists between swelling degree and solution $\mathrm{pH}$ (Table S5), but there is no systematic variation with $I$. At constant $\mathrm{pH}=4.5$ the swelling degree depends strongly on the ionic strength. By contrast, for buffers with constant ionic strength the swelling degree is practically independent of the $\mathrm{pH}$ of the solution.

Table S5 Enthalpy and entropy of the VTP in buffer solutions

\begin{tabular}{lll}
\hline & $\Delta H$ & $\Delta S$ \\
& $\mathrm{~J} / \mathrm{g}_{\text {dry gel }}$ & $\mathrm{J} / \mathrm{g} \mathrm{K}$ \\
\hline Phosphate buffers & $63.8 \pm 3.1$ & $0.21 \pm 0.01$ \\
Britton-Robinson buffers & & \\
$\mathrm{pH}=4.5$, various $I$ & $66.6 \pm 2.7$ & $0.22 \pm 0.01$ \\
$I=0.15 \mathrm{M}$, various $\mathrm{pH}$ & $76.8 \pm 6.0$ & $0.25 \pm 0.02$ \\
\hline
\end{tabular}

The DSC response of PNIPAM gels was also investigated in phosphate buffers (Fig. S2a) at fixed $\mathrm{pH}=4.5$ and at various ionic strengths (Fig. S2b), as well as in buffers of constant ionic strength (Fig. S2c). In phosphate buffers a systematic downward shift of the onset temperature and broadening of the phase transition is observed with increasing $\mathrm{pH}$, but there is no correlation between $T_{\text {onset }}$ and $I$ (Fig. 2, main text). In BrittonRobinson buffers at fixed $I=0.15 \mathrm{M}, T_{\text {onset }}$ shifts only slightly to lower temperatures with increasing $\mathrm{pH}$, with no visible change either in the shape of the endothermic transition peak or in the correlation between $T_{\text {onset }}$ and $\mathrm{pH}$ or $I$ (Table S5). $T_{\text {onset }}$ displays the same $I$ dependence for Britton-Robinson buffers with fixed $\mathrm{pH}$. The values of $\Delta H$ and $\Delta S$ are close to those in $\mathrm{KCl}$ solutions (Table 2). It can be concluded that the swelling properties are affected not only by the $\mathrm{pH}$ set by a buffer but also by the composition of the buffer. Setting the $\mathrm{pH}$, however, also changes the ionic strength of the solvent. These findings highlight the importance of the background electrolyte. 